\title{
A novel case of multiple variations in the brachial plexus with the middle trunk originating from the $\mathrm{C} 7$ and $\mathrm{C} 8$
}

Seiichi Kimura, Hiromu Amatani, Hikaru Nakai, Ren

Miyauchi, Tomohito Nagaoka, Mikiko Abe, Masatake Kai, Toshiyuki Yamagishi \& Yuji Nakajima

\begin{tabular}{|c|l|}
\hline Citation & Anatomical Science International. 95(4); 559-563 \\
\hline Issue Date & $2020-09$ \\
\hline Published & $2020-04-24$ \\
\hline Type & Journal Article \\
\hline Textversion & Author \\
\hline Rights & $\begin{array}{l}\text { This is a post-peer-review, pre-copyedit version of an article published in } \\
\text { Anatomical Science International. The final authenticated version is available } \\
\text { online at: https://doi.org/10.1007/s12565-020-00541-3. } \\
\text { See Springer Nature terms of reuse. } \\
\text { https://www.springer.com/gp/open-access/publication-policies/aam-terms-of-use. }\end{array}$ \\
\hline $\begin{array}{c}\text { Electronic } \\
\text { supplementary } \\
\text { material }\end{array}$ & $\begin{array}{l}\text { Electronic supplementary material is available online at: } \\
\text { https://doi.org/10.1007/s12565-020-00541-3. }\end{array}$ \\
\hline DOI & \begin{tabular}{l}
$10.1007 / s 12565-020-00541-3$ \\
\hline
\end{tabular}
\end{tabular}

\section{Self-Archiving by Author(s)}

Placed on: Osaka City University Repository 
A novel case of multiple variations in the brachial plexus with the middle trunk originating from the

\section{C7 and C8}

Seiichi Kimura ${ }^{1}$, Hiromu Amatani ${ }^{1}$, Hikaru Nakai ${ }^{1}$, Ren Miyauchi ${ }^{1}$, Tomohito Nagaoka ${ }^{2}$, Mikiko Abe ${ }^{1,3}$,

Masatake $\mathrm{Kai}^{1,3 *}$, Toshiyuki Yamagishi ${ }^{1,3 *}$ and Yuji Nakajima ${ }^{1,3}$

${ }^{1}$ Faculty of Medicine, Osaka City University, Osaka, Japan

${ }^{2}$ Department of Anatomy, St. Marianna University School of Medicine, Kawasaki, Kanagawa, Japan

${ }^{3}$ Department of Anatomy and Cell Biology, Graduate School of Medicine, Osaka City University, Osaka, Japan

* corresponding authors

1-4-3 Asahi-machi, Abeno-ku, Osaka-shi, Osaka, 545-8585, Japan

toshiya@med.osaka-cu.ac.jp

kai@med.osaka-cu.ac.jp

+81666453706

\section{Running title}

Multiple variations in brachial plexus 


\section{Abstract}

The brachial plexus is an important nervous structure from which all major nerves to the upper limb arise.

It typically originates from the anterior rami of the C5 to T1 spinal nerves. As it passes laterally, the roots are successively organized into three trunks, six divisions and three cords. The BP is susceptible to injury during the perinatal and postnatal periods, as well as in adulthood. Its structure can show considerable variation, and there is a wealth of literature describing its variations, providing indispensable information to neurosurgeons. Here, we report a novel unilateral variant of the brachial plexus found in an adult Japanese male cadaver. In this case, the middle trunk arose from the C7 and C8 spinal nerves, and the inferior trunk continued from the $\mathrm{T} 1$ alone. At the interscalene triangle, the subclavian artery was situated between the $\mathrm{C} 8$ and $\mathrm{T} 1$ nerves. The posterior cord arose from the posterior divisions of the superior and middle trunks, while the root from the T1 nerve/inferior trunk was absent. The anterior division of the middle trunk gave independent roots to the musculocutaneous and median nerves, without completely establishing the lateral cord. A communicating branch arose from the musculocutaneous nerve to join the median nerve. Some branches from the roots and cords also deviated from typical configurations. This case represents a rare combination of variations in the trunks, divisions, cords, and the median nerve and offers a valuable addition to the literature regarding variations in the brachial plexus.

\section{Keywords}

brachial plexus, cords, median nerve, trunks, variation 


\section{Introduction}

The brachial plexus (BP) is a complex somatic plexus comprising the origin of all major nerves innervating the upper limb. It originates in the neck and progress distally to enter the axilla with the roots, trunks, divisions, and cord regions differentiating during progression. The roots normally comprise the anterior rami of the spinal nerves $\mathrm{C} 5$ to $\mathrm{T} 1$ and the three trunks originate from the five roots: the $\mathrm{C} 5$ and C6 roots unite to create the superior trunk, C7 root continues to create the middle trunk, and C8 and T1 roots combine producing the inferior trunk. The trunks then divide into anterior and posterior divisions, which are arranged as three cords: the lateral cord originates from the anterior divisions of the superior and middle trunks, medial cord is a continuation of the anterior division of the inferior trunk, and the origin of the posterior cord is the union of all three posterior divisions. The musculocutaneous, median, axillary, radial, and ulnar nerves represent the terminal nerves of the BP. Other nerves innervating the upper limb arise from the BP at the level of the root, trunk, and cord (Standring 2016).

BP anatomy is variable, and numerous studies have described these variations since the 19th century. These include extensive works with descriptions of over 100 BPs, for example, Kerr (1918) including 175 BPs of American subjects, Hirasawa (1931) including 200 BPs of Japanese subjects, and more recently, Uysal et al. (2003), including 200 fetal BPs from Turkish subjects. Injury to the BP may occur due to disease, over-stretching, or injury, and it is commonly observed in subjects from the perinatal period up to adulthood. Studies on BP variations provide valuable information to neurosurgeons who operate in the area of this structure. 
In this case report, we present a previously undescribed BP variant, which exhibits rare

variations in the configurations of the trunks, divisions, cords, and median nerve.

\section{Case report}

We encountered a novel variant of the BP while examining the cadaver of an 86-year-old Japanese male.

The variations were unilateral; only the right side exhibited significant deviation from normal BP

architecture. A family member of the subject testified that the individual was right-handed and that no

signs of deficiency in locomotion had been observed. Atypical configurations of the trunks, divisions,

cords, and median nerve were observed amongst other variations of the BP. The variant BP comprised the

normal five roots derived from the ventral rami of the spinal nerves, C5 to T1 (Fig. 1a, b). At the

interscalene triangle, the subclavian artery ran between the C8 and T1 (Fig. 1a). The two branches from

the root regions - the dorsal scapular and long thoracic nerves-originated from the C5 and C6 nerves,

respectively (Fig. 1g; branches 1 and 2, Suppl. Fig. 1). The latter appeared to be the only nerve to innervate the serratus anterior (Suppl. Fig. 1).

Examining the lateral progress of the $\mathrm{BP}$, the roots united to form three trunks in a non-classical manner; the superior trunk constituted the C5 and C6 nerves, whereas the middle trunk originated from not only the $\mathrm{C} 7$ but from union of the $\mathrm{C} 7$ and $\mathrm{C} 8$ nerves (Fig. 1b). The $\mathrm{T} 1 \mathrm{did}$ not communicate with other roots, thus the inferior trunk was not clearly defined (Fig. 1b). The superior trunk provided the root to the suprascapular nerve (Fig. 1b, g; branch 3). The superior and middle trunks 
divided into the anterior and posterior divisions, while the T1/inferior trunk did not undergo such division.

The lateral cord was not formed completely as the anterior division of the middle trunk divided prior to meeting the anterior division of the superior trunk, resulting in separate roots of the musculocutaneous and median nerves (Fig. 1b, c). Furthermore, the posterior cord stemmed from the posterior divisions of the superior and middle trunks only, with no apparent contribution from the T1/inferior trunk (Fig. 1b). The medial cord continued directly from the T1/inferior trunk.

Anomalies of the branches of the cords were noted; the lateral and medial pectoral nerves were not readily identifiable, and the pectoralis major was innervated by two branches arising from the middle trunk and the anterior division of the superior trunk (Fig. 1b, d, and g; branches 4' and 4). The branch of the pectoralis minor did not originate from the medial cord (direct continuation of the T1), but from the lateral root of the median nerve (derived from the middle trunk, which should contain fibers of the C8 nerve) (Fig. 1b, d, and g; branch 5). The superior subscapular nerve arose from the posterior cord (Fig. 1e, $\mathrm{g}$; branch 7). The inferior subscapular and thoracodorsal nerves also stemmed from the same cord, although they emerged distally to the radial nerve (Fig. 1e, g; branches $8,8^{\prime}$, and 6). The inferior subscapular nerves exhibited three branches, one of which innervated the latissimus dorsi in addition to the subscapularis and teres major (Fig. 1e, g; branches $8,8^{\prime}$ ). The medial cutaneous nerve (of arm and forearm) originated from the medial cord as a single stem (Fig. 1b, c, and g; branch 9). Close examination revealed a short branch proceeding distally to the medial cutaneous nerve and running parallel to the ulnar 
nerve before joining it to form a small loop (omitted in Fig. 1g).

Regarding the terminal nerves of the BP, the axillary, radial, and ulnar nerves originated from the posterior, posterior, and medial cords, respectively (Fig. 1c), showing typical configuration and correct innervation. As described, the musculocutaneous nerve arose from the roots of the anterior divisions of the superior and middle trunks. The median nerve, initially created by the union of roots of the medial cord and the anterior division of the middle trunk, also exhibited an additional anastomotic branch emerging from the musculocutaneous nerve after its perforation of the coracobrachialis muscle (Fig. 1f).

\section{Discussion}

Variations in the BP are common, and understanding these variations is vital for neurosurgeons who operate in the area of the BP. Uysal et al. (2003) examined 200 BPs of spontaneously aborted fetuses and found only $46.5 \%$ had no variations.

Communication between the musculocutaneous and median nerves is a relatively frequent

variation. In the present case, the communicating branch from the musculocutaneous nerve arose between the muscular branches to the coracobrachialis and biceps brachii; we did not identify any transposition of branches from the musculocutaneous to the median nerves. According to the classifications proposed by Maeda et al. (2009) and Hayashi et al. (2017), our case is categorized as Type A2d (estimated 2.5\%) and Type II (3.1\%), respectively. 
Our dissection revealed that the long thoracic nerve originated solely from the C6 nerve

(Suppl. Fig. 1). It is normally composed of two or more roots from the $\mathrm{C} 4$ to $\mathrm{C} 7$ (with the $\mathrm{C} 6$ being the major component); this report is a rare case not described in previous studies on the structure of the long thoracic nerve by Wang et al. (2008; 38 specimens) and Nasu et al. (2012; 10 specimens).

As for the architecture of the trunks, apart from the contributions of the C4 ("prefixed") or T2 ("postfixed") nerves, the configurations of the roots are considered to be fairly consistent. Indeed, Kerr (1918) reported that the intermediate (middle) trunk was invariably formed from the C7 alone, from their study involving 175 BPs from American subjects of mixed race and gender. Meanwhile, in his investigation of $200 \mathrm{BPs}$ from Japanese subjects, Hirasawa (1931) found that $2.00 \pm 0.99 \%$ (or $2.80 \pm$ $1.38 \%$ among males) cases exhibited a middle trunk created from fusion of the $\mathrm{C} 7$ and $\mathrm{C} 8$ nerves and an inferior trunk exclusively derived from the $\mathrm{T} 1$ nerve. The author classified this novel variation as the "sixth form" and further subdivided this into four types. Arakawa (1952) observed the same class of variation occurring in $1.20 \pm 0.69 \%$ of 250 Japanese subjects. There have also been reports of this variation in Japan (Yoshida 1958; bilateral) and India (Shetty et al. 2011). In these cases, where described, an anastomosis branch from the T1/inferior trunk is observed to join the posterior trunk or the radial nerve, which is in contrast with our observations as we did not find any contribution of the T1/inferior trunk to the posterior cord. This should not compromise the function of the posterior cord because, although the inferior trunk normally provides the root of the posterior cord, few fibers from the T1 may contribute (Jenkins 2008). We found the diameter of the right T1 to be larger than that of the normal side 
(Supplementary table 1). The diameters of the anterior and posterior roots of the spinal nerves in the cervicothoracic spine appear to show positive correlations with the numbers of rootlets emerging from the anterior and posterior sulci of the spinal cord (Zhong et al. 2017). Therefore, the larger diameter of T1 in the variant BP may indicate an increased number of the rootlets, compensated from the flanking levels of spinal cord that normally correspond to $\mathrm{C} 8$ and/or $\mathrm{T} 2$. We also noted weak connecting fibers between $\mathrm{C} 8$ and T1 near the intervertebral foramen (Suppl. Fig. 2), although their significance is not clear at this stage.

The organization of the trunks that we observed appears to be uncommon in other animals (Miller, 1934), implying that it has no evolutionary origin. Convergence of the motor neurons during plexus formation is considered to be mediated in part by spatial restriction to axonal migration imposed by interactions of the Eph receptors and ephrins (Araujo et al. 1998). As the variations were unilateral in the present case, genetic causes as well as mechanical or environmental constraints during BP development may have contributed to the variations. In this regard, the existence of the subclavian artery between the $\mathrm{C} 8$ and $\mathrm{T} 1$ is worth considering. In humans, BP formation occurs at the Carnegie stage 14 (Shinohara et al. 1990), coinciding with the entrance of the axial vessel (which originates from the aortic arch and represents the subclavian artery) into the limb bud (Rodrígez-Niedenführ et al. 2001).

The correlations between the variations of the trunks, divisions, cords and the median nerve in the present case are not evident from the literature, and they may well be independent. Yet, here we propose a plausible scenario that led to these variations during morphogenesis. First, the middle trunk was 
formed from the $\mathrm{C} 7$ and $\mathrm{C} 8$ nerves as a result of mechanical constraints imposed by the subclavian artery.

Consequently, the inferior trunk was not recruited to the posterior cord as it carried only the fibers from the T1. The musculocutaneous nerve typically comprises the C5-C7 nerves, therefore the anomalous C8 fibers within the middle trunk was not attracted to it. As a result, the anterior division of the middle trunk did not unite with the anterior division of the superior trunk as a whole to create the lateral cord with the C5-C8 components; it instead branched into two to create the medial root of the musculocutaneous nerve (C7 only) and the lateral root of the median nerve $(\mathrm{C} 7+\mathrm{C} 8)$. The former gave the musculocutaneous nerve with the anterior division of the superior trunk $(\mathrm{C} 5+\mathrm{C} 6)$, while the latter combined with the medial root arising from the inferior trunk (T1). Thus the initial part of the median nerve (typically C6-T1) lacked fibers from the $\mathrm{C} 6$ nerve, which might have been supplied from the communicating branch of the musculocutaneous. This would essentially function as a second lateral root to the median nerve. Microscopic tracking of the nerve fibers may provide insight to confirm these hypotheses.

In conclusion, the BP variant reported here represents a compound of rare variations in the configuration of the trunks, divisions, cords, and median nerve, and will provide a valuable addition to the literature.

\section{Acknowledgements}

The authors wish to thank Ms Mayu Narematsu and Ms Kanako Fukuda for their technical assistance during specimen preparation. 


\section{Disclosures}

Conflict of Interest: The authors declare that they have no conflict of interest.

The materials and methods of this study did not require approval by the ethics committee of our institute.

The subject and family gave informed consent to the research, and their anonymity is preserved. 


\section{References}

Arakawa H (1952) Zum Plexus brachialis der Japaner. MIE Med J 3:107-148

Araujo M, Piedra ME, Herrera MT, Ros MA, Nieto MA (1998). The expression and regulation of chick EphA7 suggests roles in limb patterning and innervation. Development 125:4195-4204

Hayashi M, Shionoya K, Hayashi S et al. (2017) A novel classification of musculocutaneous nerve variations: The relationship between the communicating branch and transposed innervation of the brachial flexors to the median nerve. Ann Anat 209:45-50

Hirasawa K (1931) Ueber den Plexus brachialis der Japaner. Die Wurzeln des Plexus brachialis. Arb a d III. Abt d. Anat Instit d Univ Kyoto, Serie A, 2:95-153

Jenkins DB (2008) Hollinshead's Functional Anatomy of the Limbs and Back (9th edition). Saunders,

Philadelphia

Kerr AT (1918) The brachial plexus of nerves in man, the variations in its formation and branches. Am J Anat 23:285-395

Maeda S, Kawai K, Koizumi M et al. (2009) Morphological study of the communication between the musculocutaneous and median nerves. Anat Sci Int 84:34-40

Miller RA (1934) Comparative studies upon the morphology and distribution of the brachial plexus. Am J Anat 54:143-175

Nasu H, Yamaguchi K, Nimura A, Akita K (2012) An anatomic study of structure and innervation of the serratus anterior muscle. Surg Radiol Anat 34:921-928 
Rodríguez-Niedenführ M, Burton GJ, Deu J, Sañudo JR (2001) Development of the arterial pattern in the upper limb of staged human embryos: normal development and anatomic variations. J Anat 199:407-417

Standring S (2016) Gray's Anatomy: The Anatomical Basis of Clinical Practice (41st edition). Elsevier Limited, Amsterdam

Shetty SD, Nayak BS, Madahv V, Braganza CS, Somayaji SN (2011) A study on the variations in the formation of the trunks of brachial plexus. Int J Morphol 29:555-558

Shinohara H, Naora H, Hashimoto R, Hatta T, Tanaka O (1990). Development of the innervation pattern in the upper limb of staged human embryos. Acta Anat 138:265-269

Uysal II, Seker M, Karabulut AK, Büyükmumcu M, Ziylan T (2003) Brachial plexus variations in human fetuses. Neurosurgery 53:676-684

Yoshida M, Kuramoto T, Arakawa H (1958) Wanshinkeisou no mezurashii hakaku rei. Mie Kenritsu Daigaku Igakubu Kaibougaku Kyoshitsu Gyoseki Shu 9:99-102 (in Japanese)

Wang JF, Dang RS, Wang D, Zhang ZY et al. (2008) Observation and measurements of long thoracic nerve: a cadaver study and clinical consideration. Surg Radiol Anat 30:569-573

Zhong LY, Wang AP, Hong L et al. (2017) Microanatomy of the brachial plexus roots and its clinical significance. Surg Radiol Anat 39:601-610 


\section{Figure legends}

Figure 1. (a) The variant brachial plexus at the right interscalene triangle. The subclavian artery inserted between the $\mathrm{C} 8$ and $\mathrm{T} 1$ nerves. (b) The roots, trunks and cords of the variant brachial plexus. The middle trunk originated from the $\mathrm{C} 7$ and $\mathrm{C} 8$ nerves. The $\mathrm{T} 1$ nerve did not communicate with other spinal nerves nor send a root to the posterior cord. (c) The middle trunk divided into the anterior and posterior divisions, and the posterior division (arrow) provided the root to the posterior cord. The anterior division underwent further division resulting in separate roots of the median (triangle) and musculocutaneous (square) nerves. (d) Branches of the pectoralis major and pectoralis minor muscles. The pectoralis major (reflected) was innervated by branches from the anterior divisions of the superior (4) and middle (4') trunks. A branch of the medial root to the median nerve (5) innervated the pectoralis minor (reflected). (e) Branches of the posterior cord. The inferior subscapular (8 and 8') and thoracodorsal (6) nerves emerged distally to the radial nerve. One branch of the inferior subscapular nerve (8') innervated the subscapular, teres major as well as latissimus dorsi muscles. (f) A communicating branch (asterisk) arose from the musculocutaneous nerve after its perforation of the coracobrachialis muscle. (g) Schematic drawing illustrating the configuration of the variant brachial plexus. 1, dorsal scapular nerve; 2, long thoracic nerve; 3, suprascapular nerve, 4 and 4', branches to the pectoralis major; 5 , branch to the pectoralis minor;

6 , thoracodorsal nerve; 7 , superior subscapular nerve; 8 and 8 ', inferior subscapular nerves ( $8^{\prime}$ also innervates the latissimus dorsi); 9, medial cutaneous nerve (of arm and forearm); AA, axillary artery; AN, axillary nerve; BBM, biceps brachii; BM, brachialis; CBM, coracobrachialis; I, inferior; L, lateral; LDM, 
latissimus dorsi; M, medial; MCN, musculocutaneous nerve; MN, median nerve; MT, middle trunk; PC, posterior cord; PMM, pectoralis major; PmM, pectoralis minor; RN, radial nerve; S, superior; SAM, scalenus anterior; SCA, subclavian artery; SSM, subscapularis; ST, superior trunk; TMM, teres major; $\mathrm{UN}$, ulnar nerve 

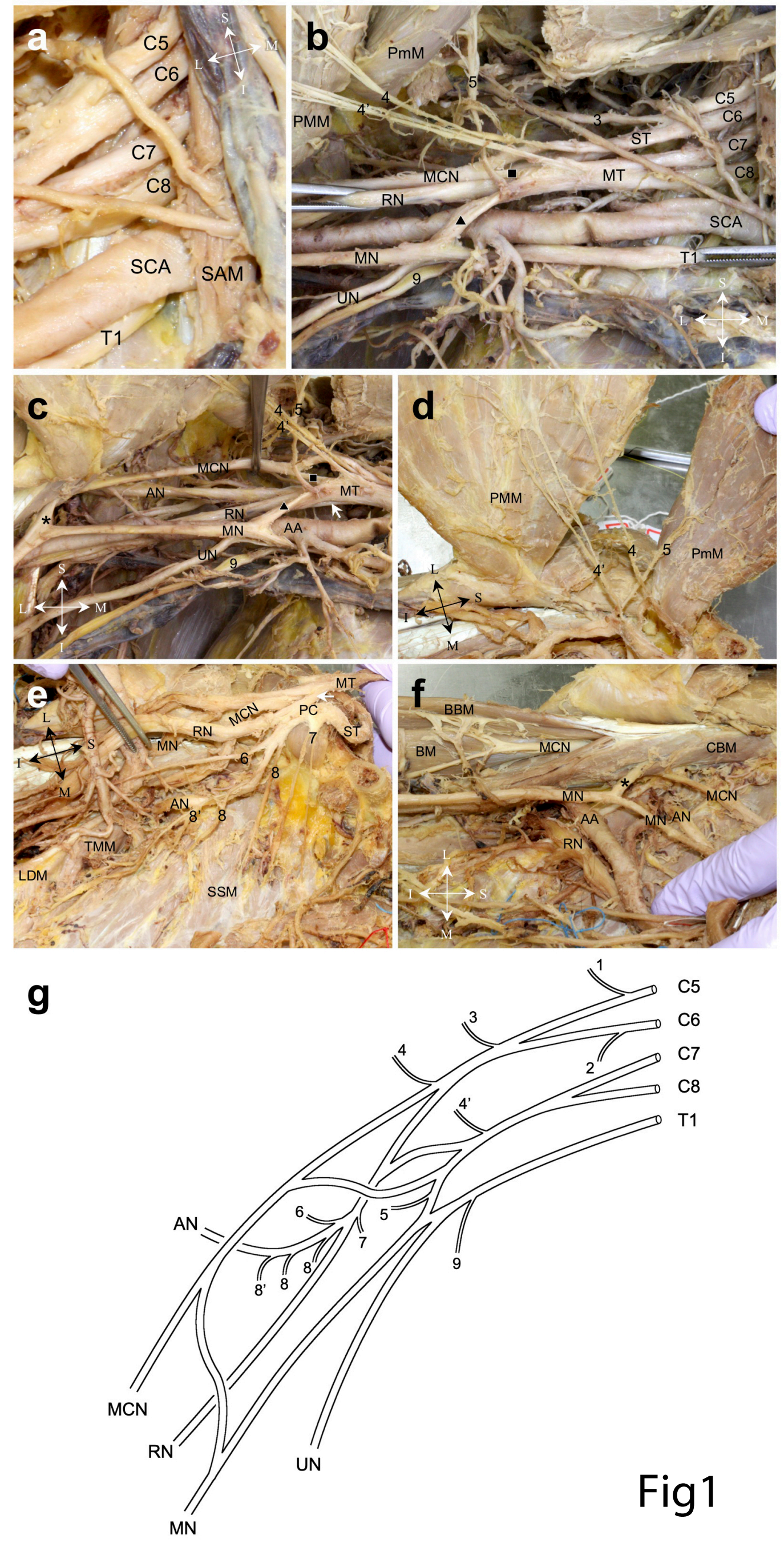


\section{Electronic supplementary material}

\section{Supplementary Materials and Methods}

Measurement of nerve fibers

The points of the largest diameter of roots and trunks were visually selected and measured using calipers.

Each point was independently measured five times, by three to five researchers. The largest and smallest figures were discarded and the remaining three measurements were used for analysis. 


\section{Supplementary Figure Legends}

Supplementary Figure 1. The long thoracic nerve (2) originated from the C6 nerve alone and innervated the serratus anterior muscle (SAM). I, inferior; L, lateral; M, medial; S, superior 


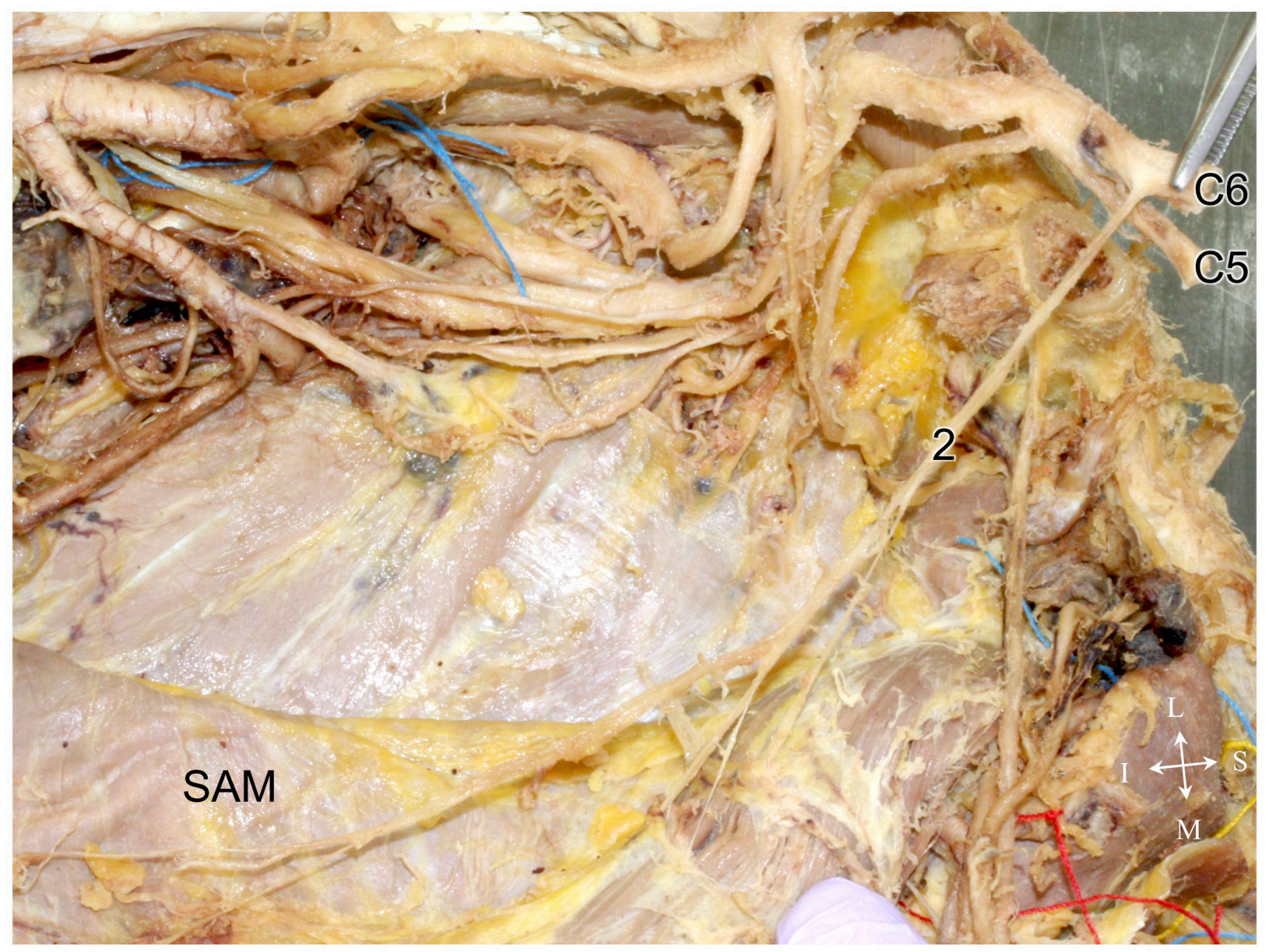

\section{Suppl_Fig1}


Supplementary Figure 2. Dorsal view of the C8 and T1 roots on the variation side. Part of the vertebral arch was removed for observation. Two weak fibers (asterisks) were found to connect the C8 and T1 nerves near the intervertebral foramen. DRG, dorsal root ganglion; I, inferior; L, lateral; M, medial; S, superior; SDM, spinal dura mater 


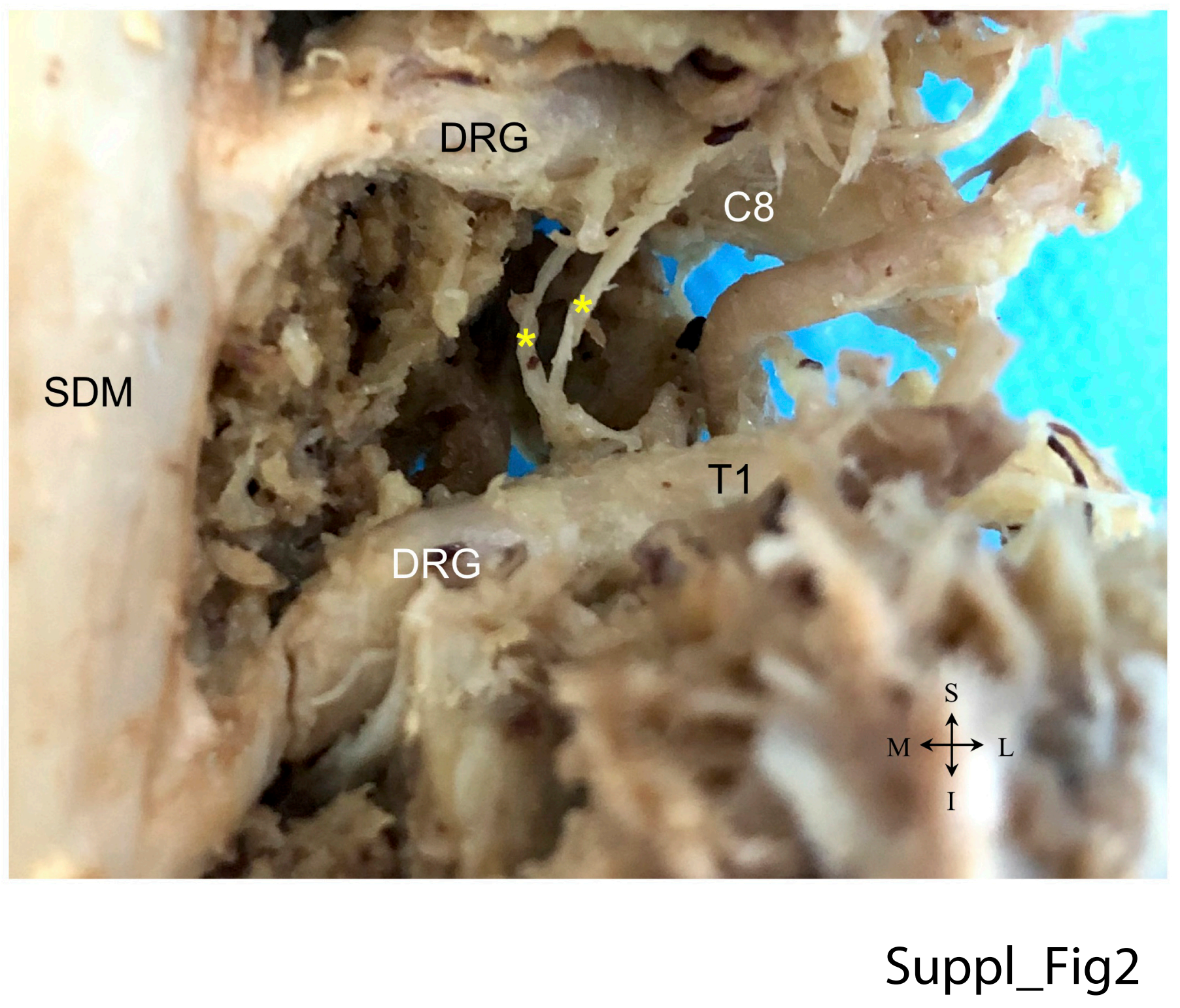




\section{Supplementary Table 1}

The largest diameter of the right and left roots and trunks

\begin{tabular}{|c|c|c|c|c|c|c|c|c|}
\hline \multicolumn{6}{|c|}{ Roots } & \multicolumn{3}{|l|}{ Trunks } \\
\hline & $\mathrm{C} 5$ & C6 & $\mathrm{C} 7$ & $\mathrm{C} 8$ & $\mathrm{~T} 1$ & Superior & Middle & Inferior \\
\hline $\mathrm{R}$ & $4.80 \pm 0.09$ & $5.63 \pm 0.06$ & $5.42 \pm 0.62$ & $5.00 \pm 0.19$ & $5.06 \pm 0.15$ & $7.40 \pm 0.27$ & $7.05 \pm 0.49$ & $6.95 \pm 0.45$ \\
\hline $\mathrm{L}$ & $6.02 \pm 0.49$ & $6.09 \pm 0.06$ & $6.20 \pm 0.20$ & $5.11 \pm 0.44$ & $4.54 \pm 0.44$ & $8.44 \pm 0.64$ & $4.88 \pm 0.72$ & $8.52 \pm 0.42$ \\
\hline
\end{tabular}

Data are presented as mean \pm standard deviation $(\mathrm{mm})$. L, left; R, right 